\title{
A importância do diagnóstico precoce na neoplasia de ovário: o papel do enfermeiro nas ações preventivas
}

\section{The importance of early diagnosis in ovarian neoplasia: the role of the nurse in preventive actions}

\author{
Rosangela Sakman' • Aline Voltarelli ${ }^{2}$
}

\begin{abstract}
RESUMO
Introdução: A área da saúde da mulher, inserida em um contexto de saúde pública, também vem sendo ameaçada por diversos agravos, destacando-se entre estes, os tumores dos ovários em virtude do diagnóstico precoce ser geralmente dificultado. Objetivo: Levantar os recursos preventivos para a identificação precoce do câncer de ovário. Método:Tratase de um estudo exploratório de revisão da literatura de abordagem qualitativa, realizado nas bases de dados LILACS, BDENF e SCIELO (Scientific Eletronic Library Online), adotando um recorte temporal das publicações entre 2005 a 2016. Resultados e Discussão: Foram selecionados 12 artigos que mostraram que, embora tenham ocorridos avanços significativos nas áreas tecnológicas e científicas, o rastreio eficaz do câncer de ovário ainda é bastante complicado. Por ser assintomático e inexistir recurso diagnóstico especifico, alguns autores apontam os fatores de riscos com meios para a investigação do câncer de ovário. Nessa proposta as mulheres são categorizadas em grupos de risco, possibilitando que a suspeita seja confirmada com a criteriosa análise de exames laboratoriais, exames de imagem e exame físico. Considera-se a história familiar o fator de risco para câncer de ovário independente mais relevante. Conclusão: As pesquisas conduzidas na última década vêm mostrando que a investigação dos fatores de risco, principalmente a predisposição familiar, é útil frente a suspeita do câncer de ovário. Nesse sentido, propõe-se relacionar malignidade para as mulheres que se enquadram nos grupos de risco, permitindo desse modo que a suspeita seja confirmada com a minuciosa análise de exames laboratoriais, exames de imagem e exame físico.

Palavras-chaves: Saúde da Mulher; Câncer de Ovário; Diagnóstico.
\end{abstract}

\begin{abstract}
Electronic Library Online) databases, adopting a temporal cut of the publications between 2005 and 2016 . Results and Discussion: selected 12 articles that have shown that although significant advances have been made in technological and scientific areas, effective screening for ovarian cancer is still quite complicated. Because it is asymptomatic and there is no specific diagnostic resource, some authors point out the risk factors with means for the investigation of ovarian cancer. In this proposal women are categorized into groups at risk, allowing the suspicion to be confirmed with the careful analysis of laboratory tests, imaging and physical examination. Family history is considered the most relevant independent risk factor for ovarian cancer. CONCLUSIONS: Research conducted over the last decade has shown that the investigation of risk factors, especially familial predisposition, is useful in the face of suspected ovarian cancer. In this sense, it is proposed to relate malignancy to the women who fit into the risk groups, thus allowing the suspicion to be confirmed by the thorough analysis of laboratory tests, imaging tests and physical examination.
\end{abstract}

Keywords:Women's Health; Ovary cancer; Diagnosis.

\footnotetext{
'Mestre em enfermagem pela Universidade de Guarulhos (UNG), especialista em saúde Pública com ênfase em saúde coletiva pela (UNINOVE), especialista em docência em enfermagem nível técnico e superior pela Faculdade Brasil, Docente pela Faculdade FAMA- UNESP e Docente pela Faculdade Sequencial. Rua: Cândida de Carvalho III,Jardim São Sebastião-São Miguel Paulista. mail: rosangelasakman @yahoo.com.br

${ }^{2}$ Mestre. Mestre. Graduada em Enfermagem pela Universidade Nove de Julho (UNINOVE); Especialização em Enfermagem do Trabalho pela Universidade Cruzeiro do Sul (UNICSUL)); Especialização em Docência em Enfermagem Nível Técnico e Superior pela Faculdade do Campo Limpo Paulista (FACCAMP), Docente convidada para pós graduação em Enfermagem na Universidade Nove de Julho, Especialização em Metodologia de Ensino a Distância pela Faculdade Anhanguera do Taboão da Serra; Docente da graduação de Enfermagem da Universidade Brasil polo FAMA de Mauá; Mestre em Ciências da Saúde pelo IASMPE. End. Rua Michael Bispo de Oliveira 247 Bairro Votupoca-Barueri - SP Brasil_mail: alivolter@yahoo.com.br
} 


\section{INTRODUÇÃO}

Nas últimas décadas, diversos são os agravos que desequilibram a saúde pública mundial. Cada área ou especialidade enfrenta problemas específicos que vão desde endemias controláveis a enfermidades ou alterações incuráveis, imprevisíveis e de difícil controle. A área da saúde da mulher, inserida em um contexto de saúde pública, também vem sendo ameaçada por diversos agravos, destacando-se entre estes, os tumores dos ovários.

As evidências acumuladas na literatura mostraram que todas as mulheres são suscetíveis a desenvolverem o câncer de ovário. Ao contrário de outros cânceres, tanto os países em desenvolvimento como os já desenvolvidos são igualmente afetados pela doença. Por conta desse cenário epidemiológico, considera-se importante que os profissionais da área de saúde possuam conhecimento sobre os sintomas, bem como dos fatores de risco e dos antecedentes familiares tanto do lado materno quanto paterno'.

Um dado preocupante a ressaltar se refere ao fato do diagnóstico precoce do câncer de ovário geralmente ser dificultado, em virtude das suas manifestações clínicas tornarem-se aparentes somente nos estágios mais avançados da doença, ou seja, quando já ocorreu a disseminação da doença pela pelve ou abdômen superior ou mesmo para outros órgãos. Constitui um câncer pouco frequente, porém com alta letalidade. A maioria dos casos não é hereditária. Portanto, no momento da descoberta da doença grande parte das mulheres acometidas encontra-se em estágios avançados.

Necessário se faz esclarecer que os tumores decorrentes do ovário são carcinomas epiteliais que ocorrem nas células da superfície do órgão afetado. Os mais comuns entre os tumores que afetam as mulheres são os de células germinativas que dão origem aos ovócitos. Já as neoplasias epiteliais do ovário são tumores constituídos por um ou mais tipos de epitélio e estroma em combinações variadas. Vale acrescentar que a maioria destes tumores é derivada do epitélio de revestimento do ovário².

Os outros tipos são muito raros. $O$ teratoma maduro quístico, pertencente ao grupo das neoplasias germinativas, é muito frequente e corresponde a cerca de $30 \%$ de todos os tumores do ovário.Ainda dentro do grande grupo de tumores dos cordões sexuais e do estroma, os tumores de células da granulosa são os que mais frequentemente, podem ter um comportamento agressivo, e correspondem a $1 \%$ da totalidade dos tumores do ovário ${ }^{3}$.

Convém ressaltar que o conhecimento da história genética torna-se fundamental para a prevenção, diagnóstico e tratamento das neoplasias.

Este conhecimento é especialmente relevante no carcinoma do ovário, porque poderá contribuir para a detecção em fases iniciais. Além disso, o conhecimento sobre os sinais e sintomas do câncer de ovário poderá permitir que as mulheres tivessem um diagnóstico antecipado e que a doença seja tratada mais facilmente. $O$ câncer ginecológico avançado é vivenciado de maneiras diversas. Muitas vezes, a paciente experimenta uma reação de revolta, desespero ou angústia, outras vezes, pode viver um estado de aceitação, com serenidade ${ }^{4}$.

As emoções e sentimentos são influenciados pelo meio em que estas mulheres estão inseridas. A maneira como uma mulher pode se comportar em relação a sua vida tende a ter influência.

Considerando a dificuldade enfrentada pelos profissionais da área da saúde para o diagnóstico precoce dos tumores do ovário, e a gravidade dos casos identificados em fases avançadas da doença, surgiu o interesse em recorrer à revisão da literatura no intuito de apresentar as evidências disponíveis sobre a importância da detecção deste câncer ginecológico, visando assim aprofundar o conhecimento, dando suporte para a tomada de decisões na prática assistencial e, portanto, melhorando a assistência prestada às mulheres ${ }^{5}$. Uma vez que é de suma importância o diagnostico precoce, podendo assim amenizar o sofrimento das mulheres acometidas por essa neoplasia tão letal. Levantar os recursos preventivos propostos existentes para a identificação precoce do câncer de ovário. Incentivar as mulheres quanto à importância dos exames preventivos, orientar a relevância em adotar consultas médica periódica evitando assim a descoberta tardia ${ }^{6}$.

\section{MÉTODO}

Trata-se de um estudo exploratório de revisão da literatura de abordagem qualitativa, com recorte transversal.A pesquisa bibliográfica pode, portanto, ser entendida como um processo que envolve as etapas: levantamento bibliográfico preliminar; formulação; elaboração do plano provisório do assunto; busca das fontes; leitura do material; fichamento; organização lógica do assunto; redação do texto ${ }^{7}$. A coleta dos dados foi realizada por meio de uma consulta mecânica e informatizada no banco de dados bibliográficos BIREME e incluiu os artigos indexados nas bases de dados LILACS (Literatura Latino -Americana em Ciências de Saúde), BDENF (Base de Dados Bibliográficos Especializada na Área de Enfermagem do Brasil) e SCIELO (Scientific Eletronic Library Online).

Os descritores utilizados nesta pesquisa foram consultados na lista de descritores em Ciências da Saúde (DECs), com o objetivo de utilizar as palavras-chave adequadas para a pesquisa bibliográfica. Para identificação dos artigos, foram utilizados os descritores do DeCS ${ }^{8}$ - Descritores em Ciências da Saúde: "Câncer de Ovário" and "Diagnóstico". Na etapa subsequente, foram selecionados os artigos de interesse para este estudo, considerando-se como critérios: artigos da área da saúde, ser um trabalho 
desenvolvido em âmbito nacional; ter sido publicado no período de 2005 a 2016 devido à escassez da literatura; estar disponível na íntegra em português online, abordar no resumo e/ou no título características e/ou aspectos sobre o diagnóstico precoce do câncer de ovário.

A partir dessa busca, realizou-se uma leitura exploratória que se constitui na verificação dos resumos com a finalidade de selecionar os artigos relacionados ao objeto de estudo; depois foi feita uma leitura do artigo na íntegra e posterior análise e discussão do mesmo de acordo com seus resultados e parâmetros; síntese dos resultados apresentados nas publicações e, por fim, o fichamento correspondente a cada publicação, destacando os núcleos temáticos construídos em cada artigo, com o auxílio de um instrumento criado para esse fim.

Os dados coletados foram armazenados em um banco de dados eletrônico, e os resultados serão apresentados em valores absolutos e percentuais na forma de tabelas e figuras. A amostra é composta por artigos científicos, na língua portuguesa, online, disponíveis na íntegra e que abordem a temática:"Neoplasias de ovário e a importância do diagnóstico precoce" no período compreendido de 2005 a 2018.

\section{RESULTADOS E DISCUSSÃO}

Com as buscas realizadas nas bases de dados anteriormente citadas e utilizando os descritores escolhidos, foi possível selecionar 12 artigos no período de 2005 a 2016, que tratam do diagnóstico precoce do câncer de ovário. No Quadro I são apresentadas as variáveis título, autor, periódico, ano, tipo e local de pesquisa dos estudos selecionados.

Após processo de refinamento dos periódicos, iniciou-se a classificação de acordo com o ano de publicação, abordagem metodológica e região de procedência dos estudos (Figuras I, 2, 3).

Nesse sentido, no que diz respeito ao ano de publicação, a grande maioria dos periódicos foi publicada no ano de 2009, totalizando 04 (34\%) estudos. No ano de 20I5, foram publicados 02 (17\%) estudos, nos anos de 2005, 2007, 2010, 20II e 2016 foi publicado 01 (8\%) estudo respectivamente. Nos anos de 2006, 2008, 20I2, 2013 e 20I4, não foram publicados estudos. Constatou-se que a maior concentração ocorreu no Estado do Rio de Janeiro (RJ) com 06 trabalhos (50\%). Os Estados de São Paulo (SP) e Rio Grande do Sul (RS) apresentaram 02 (I7\%) estudos. Já Goiás (GO) e Brasília (DF) obtiveram OI (8\%) estudo respectivamente. Conforme evidenciado pela Figura 3, é possível observar que a grande maioria da produção científica provém da região sudeste, sendo a menor produção referente às regiões sul e centro-oeste, com ausência nas regiões: nordeste e norte ${ }^{7}$.

Os autores dos estudos selecionados são unânimes em apontarem a elevada mortalidade e a quantidade mínima de casos de câncer de ovário diagnosticados em estágios iniciais, em virtude da inexistência de recursos diagnósticos para a identificação precoce 5 .

Os autores ${ }^{2,3,4}$ elucidam a utilidade da obtenção da história clínica, do exame físico, da combinação de testes diagnósticos (ecografia transvaginal e marcador tumoral CA 125) e ultrassonografia na identificação precoce do câncer de ovário ${ }^{8}$.

Informam ${ }^{12}$ que a sensibilidade do marcador tumoral CA 125 para o diagnóstico de câncer de ovário "é de $80 \%$ a $85 \%$ no tipo epitelial variando de acordo com o estadiamento, sendo de $50 \%$ no estádio I, $90 \%$ no estádio II, $92 \%$ e $94 \%$ nos estádios III e IV, respectivamente". Os estudos ${ }^{4,2,18}$ confirmam que os marcadores têm uma relevância na detecção precoce de recidiva tumoral, na triagem populacional, diagnóstico diferencial de pacientes sintomáticos, estadiamento clínico, estimativa do volume tumoral e localização de metástase. Entretanto, os autores complementam que esses marcadores devem ser associados a outros recursos para o diagnóstico, alertando que são insuficientes na detecção inicial do câncer de ovário.

Os autores enfatizam o uso da morfologia do ultrassom da escala de cinzas na caracterização de massa pélvica, distinguindo assim tumores benignos e malignos 9 .Dois estudos abordam o impacto da ooforectomia profilática sobre o risco de câncer de ovário. Lima et al. (2009) constataram que a ooforectomia profilática está indicada, em faixas etárias bem estabelecidas, para as mulheres portadoras de mutações nos genes BRCA-I e BRCA-2. Investigam os achados da literatura sobre a associação entre a oforectomia bilateral profilática e câncer de ovário em pacientes sem risco aumentado para a doença, encontrando somente um estudo que confirma o benefício da ooforectomia bilateral na diminuição do câncer de ovário ${ }^{17}$.

Estudos apontam a laparatomia mediana ampla como meio de diagnóstico, estadiamento, abordagem terapêutica do câncer de ovário em pacientes com presença de massa anexial que possuem risco de malignização. Os autores alertam que mínimas evidências referentes ao desenvolvimento do câncer de ovário são imprescindíveis na presença de uma massa anexial, em virtude do risco de malignidade. Nesse sentido, na avaliação devem ser consideradas as características aos exames de imagem, idade, história familiar, sintomatologia, achados ao exame físico e níveis dos marcadores tumorais ${ }^{16}$.

Os autores' descrevem um modelo dualístico, que agrupa os cânceres epiteliais em duas grandes categorias, designados por Tipo I e Tipo II. Os autores elucidam que ainda não foi detectada uma lesão precursora do câncer de ovário, sendo que novas teorias sobre a origem deste 
QUADRO 1 - Distribuição das publicações sobre diagnóstico precoce do câncer de ovário. Por: título, autores, ano e periódico. São Paulo, 2017.

\begin{tabular}{|c|c|c|c|c|c|}
\hline Título & Autor & Periódico & Ano & Tipo de Pesquisa & Local da Pesquisa \\
\hline $\begin{array}{l}\text { Rastreamento e diagnóstico das } \\
\text { neoplasias de ovário: papel dos } \\
\text { marcadores tumorais }\end{array}$ & $\begin{array}{l}\text { REIS, Francisco José } \\
\text { Candido dos. }\end{array}$ & $\begin{array}{l}\text { Revista } \\
\text { Brasileira de } \\
\text { Ginecologia e } \\
\text { Obstetrícia }\end{array}$ & 2005 & $\begin{array}{l}\text { Pesquisa } \\
\text { bibliográfica }\end{array}$ & Rio de Janeiro \\
\hline $\begin{array}{l}\text { Marcadores tumorais: revisão de } \\
\text { literatura }\end{array}$ & $\begin{array}{l}\text { ALMEIDA, José Ricardo } \\
\text { Chamhum et al. }\end{array}$ & $\begin{array}{l}\text { Revista } \\
\text { Brasileira } \\
\text { Cancerologia }\end{array}$ & 2007 & $\begin{array}{l}\text { Pesquisa } \\
\text { bibliográfica }\end{array}$ & Distrito Federal \\
\hline $\begin{array}{l}\text { Ultrassonografia na condução de } \\
\text { massas pélvicas }\end{array}$ & $\begin{array}{l}\text { MARTINS, Wellington de } \\
\text { Paula et al }\end{array}$ & FEMINA & 2007 & $\begin{array}{l}\text { Pesquisa } \\
\text { bibliográfica }\end{array}$ & Rio de Janeiro \\
\hline $\begin{array}{l}\text { Rastreamento e diagnóstico do } \\
\text { câncer de ovário }\end{array}$ & APPEL, Márcia et al. & Revista AMRIGS & 2009 & $\begin{array}{l}\text { Pesquisa } \\
\text { bibliográfica }\end{array}$ & Porto Alegre \\
\hline $\begin{array}{l}\text { Tumor ovariano unilateral com } \\
\text { derrame pleural: relato de caso }\end{array}$ & $\begin{array}{l}\text { FREITAS, Alexandra M. } \\
\text { S..; LONGHI, Kamila } \\
\text { Aparecida }\end{array}$ & VITTALL & 2009 & Estudo de caso & Rio Grande do Sul \\
\hline $\begin{array}{l}\text { Ooforectomia profilática: } \\
\text { indicações atuais }\end{array}$ & LIMA, Renilton Aires et al & FEMINA & 2009 & $\begin{array}{l}\text { Pesquisa } \\
\text { bibliográfica }\end{array}$ & Rio de Janeiro \\
\hline $\begin{array}{l}\text { Quando fazer ooforectomia } \\
\text { profilática com base em } \\
\text { evidências, não em suposições. }\end{array}$ & $\begin{array}{l}\text { PARENTE, Raphael } \\
\text { Câmara Medeiros et al. }\end{array}$ & FEMINA & 2009 & $\begin{array}{l}\text { Pesquisa } \\
\text { bibliográfica }\end{array}$ & Rio de Janeiro \\
\hline $\begin{array}{l}\text { Abordagem das massas anexiais } \\
\text { com suspeita de câncer de ovário }\end{array}$ & $\begin{array}{l}\text { LIMA, Renilton Aires Lima } \\
\text { et al }\end{array}$ & FEMINA & 2010 & $\begin{array}{l}\text { Pesquisa } \\
\text { bibliográfica }\end{array}$ & Rio de Janeiro \\
\hline $\begin{array}{l}\text { Marcadores tumorais de câncer de } \\
\text { ovário: o que há de novo? }\end{array}$ & $\begin{array}{l}\text { VALENTE, Vivian; } \\
\text { MASSABKI, Paulo Sergio }\end{array}$ & $\begin{array}{l}\text { Revista } \\
\text { Brasileira Clínica } \\
\text { Médica São } \\
\text { Paulo }\end{array}$ & 2011 & $\begin{array}{l}\text { Pesquisa } \\
\text { bibliográfica }\end{array}$ & São Paulo \\
\hline $\begin{array}{l}\text { Instituto Nacional do Câncer. } \\
\text { Câncer de ovário sintoma. }\end{array}$ & $\begin{array}{l}\text { Instituto nacional do } \\
\text { Câncer }\end{array}$ & $\begin{array}{l}\text { Instituo Nacional } \\
\text { do Câncer }\end{array}$ & 2012 & $\begin{array}{l}\text { Pesquisa } \\
\text { bibliográfica }\end{array}$ & São Paulo \\
\hline $\begin{array}{l}\text { Câncer no ovário ou do ovário? O } \\
\text { grande dilema atual }\end{array}$ & $\begin{array}{l}\text { ALBERNAZ Flávia } \\
\text { Renata Motta Zanoni; } \\
\text { SCHUNEMANN JÚNIOR, } \\
\text { Eduardo. }\end{array}$ & FEMINA & 2015 & $\begin{array}{l}\text { Pesquisa } \\
\text { bibliográfica }\end{array}$ & Rio de Janeiro \\
\hline $\begin{array}{l}\text { Associação de história familiar e } \\
\text { estilo de vida a morbidades em } \\
\text { pacientes com câncer de ovário }\end{array}$ & TEIXEIRA, Natália et al & $\begin{array}{l}\text { Revista } \\
\text { Associação } \\
\text { Médica Brasileira }\end{array}$ & 2015 & $\begin{array}{l}\text { Estudo de coorte } \\
\text { prospectiva }\end{array}$ & São Paulo \\
\hline $\begin{array}{l}\text { Câncer de ovário e detecção } \\
\text { precoce: revisão bibliográfica da } \\
\text { literatura }\end{array}$ & $\begin{array}{l}\text { OLIVEIRA, Katiele } \\
\text { Marques; OLIVEIRA, } \\
\text { Murielly Marques; } \\
\text { ARAÚJO, Raquel Soares. }\end{array}$ & $\begin{array}{l}\text { Revista } \\
\text { Científica } \\
\text { FACMAI }\end{array}$ & 2016 & $\begin{array}{l}\text { Pesquisa } \\
\text { bibliográfica }\end{array}$ & Goiás \\
\hline
\end{tabular}

Fonte: (LILACS, SCIELO, BDENF, 2017). 
câncer do ovário sugerem que não é no ovário que a patologia se inicia, trazendo assim novas perspectivas para o diagnóstico.

Em um estudo 6 de coorte realizado com $5 \mathrm{I}$ mulheres diagnosticadas com câncer de ovário, avaliaram a relação entre as características clínicas e demográficas com um risco aumentado para uma predisposição familiar, com base na história pessoal e familiar de câncer. Os resultados obtidos mostraram que 17 pacientes tinham uma história familiar de câncer da mama e/ou do ovário, quatro pacientes tinham uma história de câncer da mama ou do endométrio, II foram diagnosticados antes dos 50 anos, e 12 apresentaram um risco de predisposição familiar para o câncer do ovário superior a $10 \%{ }^{10}$. O diagnóstico do câncer de ovário não é fácil. No início, os sintomas são inexistentes ou muito vagos e, quando se faz o diagnóstico, a maioria dos casos já não está mais localizada nos ovários, tendo se espalhado pelo abdome ${ }^{11,26}$.

Os achados na literatura mostram que, embora tenham ocorridos avanços significativos nas áreas tecnológicas e científicas, o rastreio eficaz do câncer de ovário ainda é bastante complicado, em razão desse tipo de tumor ser assintomático no decorrer do seu processo de desenvolvimento, se manifestando clinicamente somente quando estar em um estágio avançado. Além desse agravante, não existe um método diagnóstico considerado confiável, de fácil execução e realizável em todas as mulheres ${ }^{13}$.

Assim sendo, por ser assintomático e inexistir recurso diagnóstico especifico, alguns autores apontam os fatores de riscos com meios para a investigação do câncer de ovário. Nessa proposta as mulheres são categorizadas em grupos de risco, possibilitando que a suspeita seja confirmada com a criteriosa análise de exames laboratoriais, exames de imagem e exame físico ${ }^{4,6,13}$.

Considera-se a história familiar o fator de risco para câncer de ovário independente mais relevante. As evidências mostraram que a presença de dois familiares de primeiro grau com o diagnóstico do tumor associa-se a um risco relativo de desenvolver, igual a 3,6; já entre parentes de segundo grau este mesmo risco se reduz para 2,9. Nesses casos, são descritas mutações nos genes BRCA-I e BRCA-2, localizados nos cromossomas 17 e I3, respectivamente, sendo responsáveis por $90 \%$ dos casos de câncer hereditário. O BRCA-I se responsabiliza por aproximadamente $60 \%$ dos tumores ovarianos e a mutação BRCA-2 por 30\%, 322,20 .

Estudos confirmam que os principais fatores de risco não modificáveis para o câncer de ovário são: idade, suscetibilidade genética e mulheres portadoras de mutações deletérias nos genes relacionados ao câncer de ovário ${ }^{6}$.

Uma vez identificada à população de risco recomenda-se o rastreio do câncer de ovário por meio de dosagens seriadas do marcador tumoral sérico $C A$ 125 e ecografia transvaginal, (VALENTE, MASSABKI, 20I I; REIS, 2005).

As diretrizes estabelecem que as mulheres identificadas como população de alto risco (histórico familiar positivo), a partir dos 35 anos, devem realizar a cada seis a doze meses, em combinação, a dosagem sérica de CA 125 e a ecografia transvaginal. Nos casos em que os resultados são anormais indica-se a ultrassonografia ${ }^{4,13,22}$.

Contudo, os resultados obtidos nos estudos conduzidos na última década mostraram resultados contraditórios sobre a eficácia dessa estratégia em relação à identificação precoce e redução na mortalidade 4,15 .

Por ser o marcador tumoral CA 125 uma sialomucina de elevado peso molecular, amplamente chamada de MUC 16, adota-se um ponto de corte de $35 \mathrm{U} / \mathrm{ml}$ como método para detectar 0 câncer de ovário ${ }^{16}$ enfatizam que o marcador tumoral CAI 25 encontra-se elevado apenas na metade dos tumores ovarianos em estádio I. Também advertem que níveis elevados de CA 125 (medidas acima de $35 \mathrm{Us} / \mathrm{ml}$ ) são observados em cânceres ginecológicos (trompa, endométrio e endocérvice) e no câncer de pulmão e trato gastrintestinal. Por isso, o emprego isolado desse antígeno, como método de rastreamento vem sendo amplamente discutido ${ }^{17}$. Entendese que além do valor absoluto verificado inicialmente, a idade da paciente e, principalmente, os valores obtidos em dosagens seriadas devem ser também considerados. Mulheres com carcinoma de ovário apresentam níveis de CA 125 crescentes, sendo recomendada a ecografia transvaginal, independente de resultados absolutos normais (CA 125 menor que $35 \mathrm{Us} / \mathrm{ml})^{18,23}$.

Alguns estudos mostraram que os Marcadores Tumorais associados com a ultrassonografia não possibilitam o diagnóstico precoce, contudo, auxiliam no diagnóstico e subsidiam na detecção da reincidiva tumoral ${ }^{21,25}$.

\section{CONCLUSÃO}

Os achados na literatura confirmaram que o câncer de ovário apresenta baixa prevalência, contudo, é apontado como o principal protagonista na mortalidade entre as neoplasias ginecológicas.

As evidências disponíveis mostram que o diagnóstico tardio devido à inexistência de recursos eficazes e a escassez de sintomas nas fases iniciais da doença justificam, em parte, a baixa sobrevida e significativa mortalidade.As pesquisas conduzidas na última década vêm mostrando que a investigação dos fatores de risco, principalmente a predisposição familiar, é útil frente a suspeita do câncer de ovário.

Nesse sentido, propõe-se relacionar malignidade para as mulheres que se enquadram nos grupos de risco, permitindo desse modo que a suspeita seja confirmada com 
a minuciosa análise de exames laboratoriais, exames de imagem e exame físico.Assim sendo, os profissionais da área da saúde, sobretudo da equipe de Enfermagem, precisam possuir conhecimento aprofundado sobre os fatores de risco para o câncer de ovário, por permitir a abordagem das mulheres com base em evidências científicas.

De igual modo, devem saber quais são os sintomas deste tipo de câncer ginecológico.A partir da identificação das mulheres que se enquadram na categoria de risco, torna-se possível encaminhá-la para a realização dos exames propostos na atualidade para o diagnóstico do câncer de ovário, que são as dosagens seriadas do marcador tumoral sérico CA 125 e ecografia transvaginal.É fundamental, portanto, identificar quem faz parte do grupo de pacientes com perfil de alto risco e, a partir disso, traçar a estratégia mais eficaz para a investigação diagnóstica. 


\section{REFERÊNCIAS}

I.Albernaz, FRMZ.; Schunemann Júnior, E. Câncer no Ovário ou do Ovário? O grande dilema atual. Femina. 20I5;43(4):I6773.

2.Almeida, JRC. et al. Marcadores tumorais: revisão de literatura. rev. brasileira cancerologia, 2007;53(3):305-31.

4.Appel, M.et al. Rastreamento Diagnóstico Câncer de ovário. Rev.AMRIGS. 2009;53(3):3 I3- 18

5.Freitas, MAS, longhi KA. Tumor ovariano unilateral com derrame pleural: Relato de caso.Vittalle. 2009;2 I(I):37-42..

6.Oliveira, KM.; Oliveira, MM.; Araújo, RS. Câncer de ovário e detecção precoce:revisão bibliográfica da literatura. revista científica facmais, v. 7, n. 3, p. 59-65, 2016.

7. Gil,AC. Como elaborar projetos de pesquisa. 5. ed. são paulo: atlas, 2010.

8.Biblioteca Virtual em Saúde, descritores em saúde. disponível em: http://decs.bvs.br/cgi-bin/wxis I660.exe/decsserver/ acesso em: 03/06/2018.

9.Brasil. Instituto Nacional de Câncer. Incidência de câncer no Brasil. estimativa 2016/2017. disponível em: <http://inca. gov.br>. acesso em: 22 mar. 2017.

10.Brasil. Instituto Nacional de Câncer. Câncer de ovário sintoma. disponivel em: http://inca.gov.br>.acesso em: 22 mar.2017.

I I.Cho, KR.; SHIH, IM. Ovarian Cancer. annual review pathology, n. 4, p. 287-3।3, 2009.

12.Cruz, LMB.; loureiro, RP.A comunicação na abordagem preventiva do câncer do colo do útero: importância das influências histórico-culturais. saúde sociedade, v. 17, n. 2, p. |20-|3|, 2008.

I3.Dantas, CN. et al. AConsulta de Enfermagem Câncer Cérvico-uterino para mulheres que a Vivenciaram. Rev. rene, $v$. I3, n. 3, p. 59|-600, 2012.

14. Teixeira, N. et al. Associação de história familiar e estilo de vida a comorbidades em pacientes com câncer de ovário. rev. associação médica brasileira, v. 6I , n. 3, p. 239-239, 2015.
15.Valente, v.; Massabki, PS. Marcadores tumorais de câncer de ovário: o que há de novo? rev. brasileira clínica médica São Paulo, v. 9, n. 5, p. 377-38I, 201 I.

16.Lima, RA. et al.Abordagem das massas anexiais com suspeita de câncer de ovário. femina, v. 38, n. 6, p. 259-262, 2010.

17. Parente, RCM. et al. Quando fazer ooforectomia profilática com base em evidências, não em suposições. femina, v. 37 , n. 10, p. 527-533, 2009.

18.Martins, WP. et al. Ultrassonografia na condução de massas pélvicas. femina, v. 35, n. 6, p. 345-349, 2007.

19.Melo, MCSC. et al. O enfermeiro na prevenção do câncer do colo do útero: o cotidiano da atenção primária. rev. bras. cancerologia, v. 58, n. 3, p. 389-398, 2012.

20.Miranda,D.et al.Sistema genital feminino. in: brasileiro filho, $g$. bogliolo patologia. oito ed. Rio de Janeiro: guanabara koogan, 20I I. p. 554-589.

2I.Reis, FJC. Rastreamento e diagnóstico das neoplasias de ovário: papel dos marcadores tumorais. rev. brasileira ginecologia obstetrícia, v. 27, n. 4, p.222-227, 2005.

22.Ristow, CM.; Yamamoto, CT.; fávaro, m.Fatores de risco e patogênese das neoplasias malignas epiteliais de ovário:revisão de literatura. rev. brasileira cancerologia, v. 52, n. 2, p. I85-195, 2006.

23.Rodrigues, d.; Santos,VE. A educação em saúde na estratégia saúde da família: uma revisão bibliográfica das publicações científicas no Brasil. journal health science institute, v. $28, \mathrm{n}$. 4, p. $321-324,2010$.

24.Zorzi, r.; Starling, IG. Sistema Reprodutor Feminino. in: zorzi, f. corpo humano: órgãos, sistemas, funcionamento. são paulo: senac nacional, 20I0. p. I7I - I75.

25.Watanabe, MAE. et al. Gestação: um desafio Imunológico. semina: ciências biológicas e da saúde, londrina, v. 35, n. 2 , p. I47-I62, 2014.

26.Widmaier, EP.; Raff, H.; Strang, TS. fisiologia humana: Os mecanismos das funções corporais. 9 ed. rio de janeiro: guanabara koogan, Rio de Janeiro, $201 \mathrm{l}$. 